\title{
The Accessibility Analysis of Highway Traffic from the Perspective of Regional Economic Integration -A Case Study of Nanchang-Jiujiang Region
}

\author{
Zhangling Zou ${ }^{1,2, a}$, Chaoyang FANG ${ }^{* 1,2,3, b}$, Yanjiang PAN ${ }^{1,2, c}$ \\ ${ }^{1}$ School of Geography and Environment, Jiangxi Normal University, Jiangxi, China; \\ ${ }^{2}$ Key Laboratory of Poyang Lake Wetland and Watershed Research, \\ Ministry of Education, Jiangxi, China \\ ${ }^{3}$ Key Laboratory of Watershed Ecology and Geographical Environment Monitoring, National \\ Administration of Surveying, Mapping and Geoinformation, Jiangxi, China

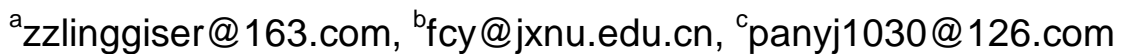

\begin{abstract}
Keywords: regional economic integration; cities' synchronization effect; traffic accessibility; Nanchang-Jiujiang Integration.

Abstract. "Nanchang-Jiujiang Integration" is an important development strategy in Jiangxi Province. This paper embarks from the perspective of promoting regional economic integration, and takes time-distance for an index, and quantitative analysis the highway traffic accessibility in Nanchang-Jiujiang region from different aspects. It aims to evaluate whether the current highway traffic infrastructure could meet the requirements of the development of strategy of "Nanchang-Jiujiang Integration". The research results are as follows: Firstly, the hour communication circles with the core of downtown Nanchang and downtown Jiujiang have covered most parts of Nanchang-Jiujiang region, Either of them can reach the other one and the cities' synchronization effect has been preliminary presented. Secondly, the entirely accessibility of Nanchang-Jiujiang region is in good condition, but there are great differences among the areas. The spatial pattern of accessibility presents an appearance of high-grade road direction and dense network direction. Thirdly, due to the obstructing effect of drainage, there are large differences in accessibility between two sides of a large body of water. Based on the results, we put forward some recommendations to enhance the level of the Nanchang-Jiujiang integration in view of the existing traffic construction and strategic planning.
\end{abstract}

\section{Introduction}

Transport is an important prerequisite and foundation for regional economic integration, which is the objective requirement of the development of cooperation among the regional economies ${ }^{[1]}$. With the deepening of Chinese political reform, and gradually establish and perfect the market economy system, Chinese regional economic development mode from "administrative regional economy" (1992, Liu Junde) transition to "regional economic integration" ${ }^{[2]}$. Traffic is the main carrier of the interregional personnel, material and other elements of flow and spatial linkages ${ }^{[3]}$, which may reduce spatial-temporal distance of economic activity on a certain way, and break the barriers to the administrative boundaries, to promote market integration. The rational planning can improve regional accessibility, improving location conditions, which is an important means to promote regional economic integration, and can directly reflect regional economic integration ${ }^{[4-5]}$. Accessibility refers to the use of a certain type of traffic from a given location to location of convenience ${ }^{[6-7]}$, it is important indicator to measure levels of transportation and construction, rationality of road network structure ${ }^{[8]}$. Improvement of regional accessibility help improving location conditions, reduce circulation costs between the regional economic entity.

" Nanchang-Jiujiang Integration" refers to the development strategy of regional economic communities implementing economic integration between Nanchang and Jiujiang cities, in Jiangxi 
province, which goal is building integration patterns to form agglomeration benefits, taking transport integration of major infrastructure construction as the main means, and leading to economic development across the province. In this Paper on the basic of multi-scholars research on regional traffic accessibility, contrapose the Nanchang-Jiujiang regional economic community, quantitative analysis on different levels of regional highway traffic accessibility, aimed at assessment existing highway traffic facilities level can satisfy the development strategy requirements of " Nanchang-Jiujiang integration".

\section{Study area and datasets}

Nanchang-Jiujiang region refers to Nanchang, Jiujiang city, which contain all the jurisdiction of counties (cities, districts), and the development zone. Nanchang is capital city of Jiangxi province, which is the political, economic, culture and traffic center of Jiangxi province, which governs four Counties: Nanchang County, Xinjian County, Anyi County and the Jinxian; Jiujiang is important of Portal city in Jiangxi province, and it administers Jiujiang, Wuning, Xiushui, Yongxiu County,, and Xingzi, De ' an, Duchang, and Hukou 8 counties and the escrow Ruichang city and Gongqing city (Figure 1). Nanchang and Jiujiang are connected by geographical space, and with close economic ties, similar geographical conditions, and concentrated developmental elements. They are provided with the formation of regional economic community, which build a good foundation for integration of two cites.

In this study data source mainly includes highway traffic data of Nanchang-Jiujiang area, basic geographic data and social economic data. Highway traffic data included highways, national roads, provincial roads, county and town roads and urban expressways, main roads, secondary roads, slip roads within the study area. All data were collected by interpreting the $8 \mathrm{~m}$ high spatial resolution remote sensing images in Nanchang-Jiujiang region which obtained in 2012 and extracted road centerline. Establishing the spatial database via add attributes of road grade, traffic speed road network, etc; the basic geographic data, such as urban node data, rivers and lakes, administrative divisions, come from the data of " Jiangxi province map set" (China Cartographic Publishing House, 2008); socio-economic data is from "Nanchang statistical Yearbook -2014" and "Jiujiang statistical Yearbook -2014" were published by China statistical Publishing House.

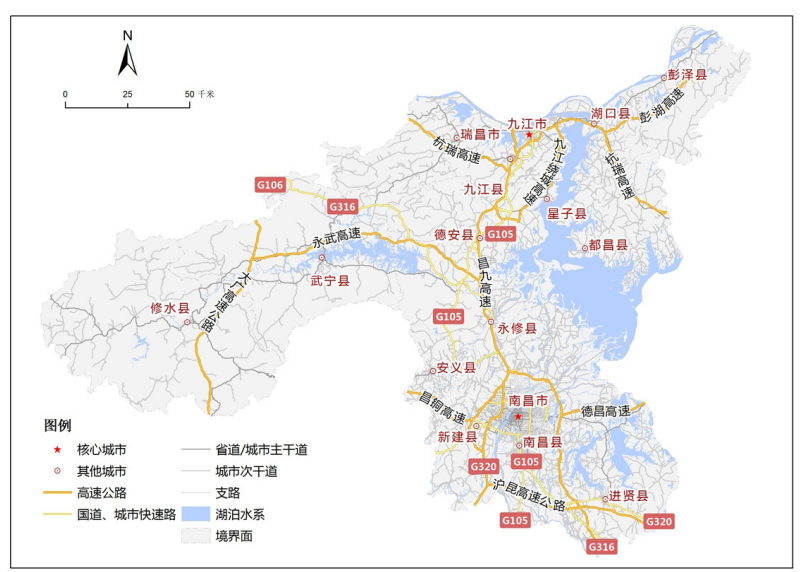

Figure.1 General Situation and the traffic network

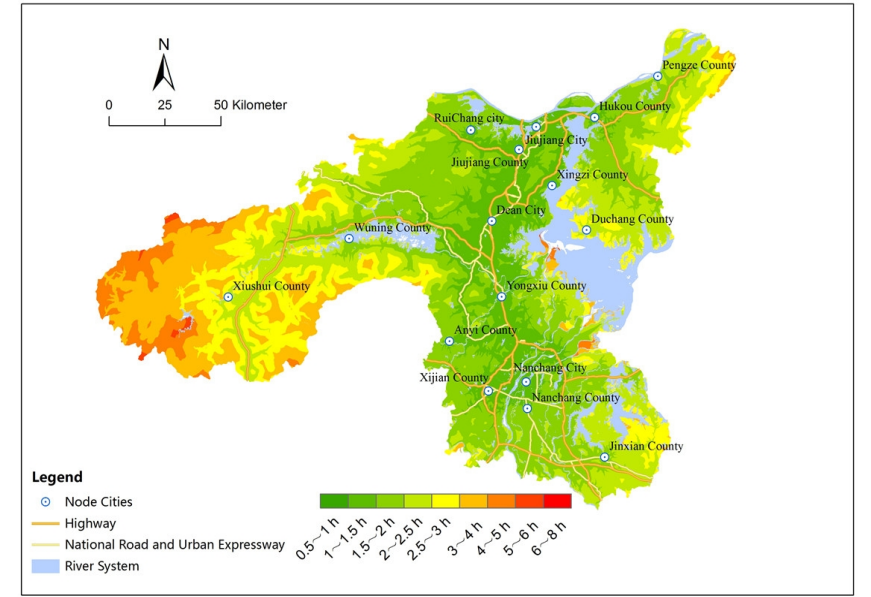

Figure.2 Spatial pattern of average accessibility in Nanchang-Jiujiang regionin Nanchang-Jiujiang region

\section{Methods}

\section{Research methods of accessibility}

In this paper, the GIS spatial analysis method is utilized to calculate the accessibility of the index with the shortest travel time. Specific research methods are as follows:

(1) The division of the grid: the study area is divided into $50 \mathrm{~m} * 50 \mathrm{~m}$ grid, the grid area of $0.0025 \mathrm{~km}^{2}$. Each grid size is small enough to be considered as the reachable mean of the grid with respect to the 
area of the study area $26225.36 \mathrm{~km}^{2}$. The study area is divided into 10490144 grids.

(2) Handle with the adjacent regional road network: in order to maintain the network integrity and continuity, improve accessibility value calculation accuracy, this paper conducted the accessibility analysis that joined the network of the area surrounding counties as a whole region, based on the construction of Nanchang -Jiujiang highway network, in the final analysis results analysis of patterns in the study area are extracted as a result. In the final, extracting the study area of analysis pattern as the result of this research.

(3) Road speed setting: according to the "the People's Republic of China highway engineering technique standard JTGB01-2003" on all levels of road design speed limits and road design traffic speed. Conducting accessibility analysis of Nanchang-Jiujiang regional highway traffic network in this paper, the highway speed set at $100 \mathrm{~km} / \mathrm{h}$, the national highway speed is set to $80 \mathrm{~km} / \mathrm{h}$, the highway speed is set to $60 \mathrm{~km} / \mathrm{h}$ and county road speed set at $40 \mathrm{~km} / \mathrm{h}$, the urban trunk road speed is set to $60 \mathrm{~km} / \mathrm{h}$ and secondary roads speed is set to $40 \mathrm{~km} / \mathrm{h}$, the urban freeway speed set at $80 \mathrm{~km} / \mathrm{h}$, branch speed set at $20 \mathrm{~km} / \mathrm{h}$, the area with no roads but can walk through is set to $5 \mathrm{~km} / \mathrm{h}$.

(4) Barrier grid processing: the grids located in rivers and lakes region are regarded as barrier grid, but the grid which belong to in the regional of barrier connected the bridge or tunnel of non-barrier region were assignment according to the actual speed; expressway are closed road, in addition to the entrances and exits of expressway, the interior 20 meters buffer of highway were act as barrier grid.

\section{Measurement index of accessibility}

This paper use the shortest travel time as accessibility measure indexes, the formula is shown below:

$$
A_{i}=\sum_{j=1}^{n} \frac{T_{i j}}{n}
$$

In the formula, $j$ is arbitrary point in the area, $i$ is the node of city, it represents administrative region. $T_{i j}$ stands for the traffic time for node $i$ get through the road network traffic time of the lowest cost route to $j$. $\mathrm{N}$ nodes for the city, that is, for the node I accessibility value, the smaller of the value that means the better accessibility on the node, and the travel of city residents is more convenient.

\section{Nanchang-Jiujiang regional traffic accessibility pattern analysis}

We treat Nanchang-Jiujiang road network as transport channels, equation (1) was used to obtained the shortest travel time in the region of 16 node cities, took the average value of the shortest travel time can acquire an average spread of accessibility of the region (Figure 2). The average regional accessibility has the capability to delineate the whole region, which represents the spatial-temporal distribution characteristics of accessibility in Nanchang-Jiujiang region.

Nanchang-Jiujiang area present T-shaped branch, from an overall perspective, in addition to edge areas, the cities and counties in the central " | " of the district are located within the 2 hour average accessibility, the accessibility level is significantly higher than in "-" region which at both ends of the counties. This is because the Nanchang-Jiujiang highway in the region focused on the " | "region, while there are less high grade roads in "-" area, especially in Xiushui County, only the Da-Guang expressway pass by, so it indicated lower level of accessibility. The accessibility of eastern of Poyang Lake wetland, Junshan Lake, Qinglan Lake, and Jinxi Lake was significantly lower than the western coast of the Lake; Zhelin Reservoir's accessibility on the West side higher than the East; due to the bridge connecting role, accessibility between the two sides of rivers in Nanchang-Jiujiang region (such as Ganjiang, Xiushui, Fuhe, etc) have no significant differences.

\section{Conclusions and Implications}

(1)As a whole the Nanchang-Jiujiang area accessibility is well, but the regional differences are large. Accessibility in vertical area is better than horizontal area, the centre area is better than the edge. 
Accessibility distribution pattern shows obvious high-grade and network density road directivity: there are more high-grade road in vertical area, and accessibility was significantly higher than the horizontal area; with density high-grade road in center area, their accessibility better than the edge area. From the perspectives of improving the level of accessibility in the entire region, the government should increase high-grade road construction of the horizontal area and edge area, increasing the density of road networks, optimize network structure.

(2) the road construction planning should be combined with tunnels, bridges and other diversity ways to improve the road network structure, complete the Nanchang-Jiujiang highway network. As multi-drainage area, Nanchang-Jiujiang areas need to strengthen the construction of bottom tunnels, across Lakes and River projects, improvement of regional accessibility differences caused by the water barrier.

" Nanchang-Jiujiang integration" strategy can not be accomplished in one day or night, it is going to be a long process, and the goal will be achieved ultimately, the propulsion and implementation of urban integration of Nanchang-Jiujiang area will promote regional industrial restructuring, promoting coordinated regional development, speeding up urbanization process and enhance regional economic development strength, which will lead Nanchang and Jiujiang entering the new era of development.

\section{Acknowledgements}

This work was financially supported by the Innovation Fund designated for Graduate Students of Jiangxi Province (NO.YC2014-S156); Special Scientific Research Fund of Surveying,Mapping and Geoinformation Public Welfare Profession of China (201512026); Director's Opening Fund of Key Laboratory of Poyang Lake Wetland and Watershed Research (Jiangxi Normal University), Ministry of Education(ZK2013004) and Collaborative Innovation Center for Major Ecological Security Issues of Jiangxi Province and Monitoring Implementation(JXS-EW-00).

\section{References}

[1] Zhiping Wang.. Economic Research, 2007, 29(12): 6-8.

[2] Junde Liu. Journal of Hangzhou Normal University,2000,(1):15-19.

[3] Yang Xu, Enchong Xi, Bin Su. theory and reform, 2013, (2): 97-99.

[4] Shenglong Liu, Aagang Hu. Economic Research, 2011(3): 72-82.

[5] Yuhong Liu, Xi Wang, Journal of Xi'an Jiaotong University,2014,34(3):43-48.

[6]KOENIG J G.. Transportation, 1980,(9):145-172.

[7]Morris J M,Dumble P L,Wigan M R. Transportation Research A, 1978, (13):19-109.

[8]Gutiérrez Javier, Ana Conde- Melhorado, Juan Carlos Martín.. Journal of Transport Geography, 2009, 12(3): $1-12$. 